\title{
Optogenetics at cellular resolution
}

A combination of restricting channelrhodopsin to the soma and restricting the excitation volume with temporal focusing enables the mapping of neural circuits with high precision.

The optogenetic manipulation of neurons has become a standard approach for neural circuit analysis since its inception more than a decade ago. Refinements in the excitation strategies, such as with two-photon illumination, have allowed more detailed analyses at the resolution of single neurons. But when single neurons are targeted, neurites from other cells in close proximity may be localized in the excitation volume, leading to unwanted excitation of other cells. McLean Bolton and her team at the Max Planck Florida Institute for Neuroscience, including first author Christopher Baker, have found a way to alleviate this concern by restricting the expression of channelrhodopsin to the soma and proximal dendrites and by shaping the two-photon excitation volume with temporal focusing.

"With two-photon excitation of conventional channelrhodopsin, you can trigger action potentials even when you are off the cell body of a neuron," cautions Baker. One obvious way to avoid optogenetic activation in passing is to exclude opsins from neurites. Baker explains that several motifs are known to be capable of restricting heterologous proteins to a somatodendritic localization. "The one that appeared to have the most promise was the C-terminus of the Kv2.1 potassium channel," says Baker. This motif had already been described by the lab of Zhuo-Hua Pan at Wayne State University. Baker and colleagues built on this finding and combined it with two-photon excitation for circuit mapping at the single-cell level.

Another key component of the precision optogenetics approach is the illumination strategy. Several methods for two-photon illumination of optogenetic tools have been reported, and they all have their benefits and drawbacks. The researchers chose to shape the excitation volume using temporal focusing. This strategy allows expansion of the excitation beam to cover the soma of a neuron while enabling the axial extent of illumination to be controlled independently. With this approach, the researchers can manipulate single cells but not ensembles of neurons at the same time. "We were eventually going to go to holography ... but we figured temporal focusing was going be the first iteration that would provide us with what we needed," explains Baker.

The researchers applied the technology to map synaptic connections of neurons in acute slices of the mouse somatosensory cortex. They evoked action potentials in neurons surrounding a cell that they recorded from in patch-clamp mode. The researchers found that about $10 \%$ of stimulated neurons induced electrical activity in the recorded cells, which is consistent with the connectivity rate observed with paired recordings. In contrast, when using channelrhodopsin that was not restricted to the soma, they observed an apparent connectivity rate of about $30 \%$, pointing to direct stimulation of the patched cell.

Baker and Bolton plan to use the somatargeting approach to look at local circuitry in acute slice preparations from animal models of psychiatric diseases. They might eventually extend the approach to in vivo applications, in collaboration with others, but for now their strategy will be useful for studying local circuits in areas in the mouse brain that are difficult to reach in in vivo preparations, such as the amygdala.

Baker is also interested in finding even better means of restricting opsins to the soma. "Kv2.1-mediated restriction isn't necessarily completely absolute, and there is in some cells expression in the proximal dendrite," explains Baker. He says that the mechanism behind the soma restriction is not fully understood, but it is possibly due to the targeting of certain vesicles to the soma, which might be mediated by certain myosin
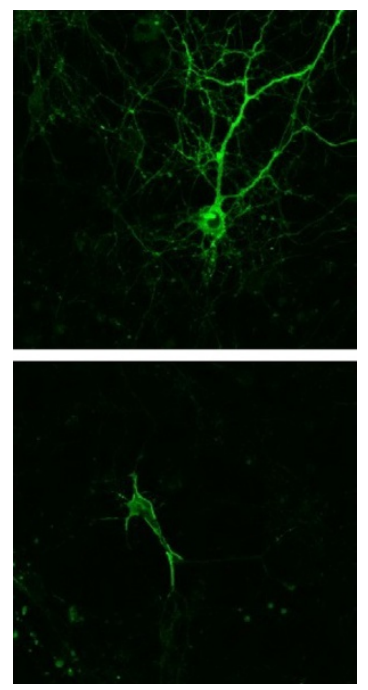

Untargeted (top) and targeted (bottom) channelrhodopsin expression in cortical neurons. Reproduced from Baker et al. (2016) (https://creativecommons.org/licenses/by/4.0/ legalcode).

motors. Other, less efficient strategies could rely on rapid endocytosis of the targeted proteins. In this case, turnover would be rapid, and there would not be enough time for the targeted proteins to spread far in the neurites. "We are thinking of trying to find things that are similar to a Kv2.1 motif that would put things into vesicles that have that kind of rather restricted somatodendritic distribution," says Baker, explaining his rationale for further improving somatic restriction of opsins.

Their current Kv2.1-motif-based strategy should be useful for other opsins as well. Baker has already tested the motif in other contexts, showing that it works with other opsin variants such as C1V1, ReaChR and Chronos. "We think that it should work with just about any opsin," says Baker.

Nina Vogt

\section{RESEARCH PAPERS}

Baker, C.A. et al. Cellular resolution circuit mapping with temporal-focused excitation of soma-targeted channelrhodospin. elife 5, e14193 (2016). 